\title{
ANTIMICROBIAL EFFECTS OF ALLICIN AND KETOCONAZOLE ON TRICHOPHYTON RUBRUM UNDER IN VITRO CONDITION
}

\section{${ }^{1}$ Farzad Aala, ${ }^{2}$ Umi Kalsom Yusuf, ${ }^{3}$ Farida Jamal, ${ }^{4}$ Sassan Rezaie*}

${ }^{1}$ Department of Medical Mycology \& Parasitology, Faculty of Medicine, Kurdistan University of Medical Sciences, Sanandaj, Kurdistan, Iran; ${ }^{2}$ Department of Biology, Faculty of Science; Universiti Putra Malaysia, 43400 Serdang, Selangor, Malaysia; ${ }^{3}$ Department of Medical Microbiology and Parasitology, Faculty of Medicine and Health Sciences; Universiti Putra Malaysia, 43400 Serdang, Selangor, Malaysia; ${ }^{4}$ Department of Medical Biotechnology, School of Advanced Technologies in Medicine, Tehran University of Medical Sciences, Tehran, Iran.

Submitted: May 04, 2011; Approved: January 16, 2012.

\begin{abstract}
Dermatophytosis is caused by a group of pathogenic fungi namely, dermatophytes, is among the most prevalent infectious diseases worldwide. Azole drugs are widely used in the treatment of dermatomycosis, but can cause various side effects and drug resistance to the patients. Hence, for solving this problem can be used from the plant extract as alternative for chemical drugs. Allicin is a pure bioactive compound isolated from garlic was tested for its potential as a treatment of dermatomycosis in this study. This study evaluated the in vitro efficacy of pure allicin against ten isolates of Trichophyton rubrum and the $\mathrm{MIC}_{50}$ and $\mathrm{MIC}_{90}$ ranged from $0.78-12.5 \mu \mathrm{g} / \mathrm{ml}$ for allicin. The results revealed that the order of efficacy based on the MICs values, all isolates showed almost comparable response to allicin and ketoconazole except for some isolates, at $28{ }^{\circ} \mathrm{C}$ for both 7 and 10 days incubation. Mann-Whitney test indicate that MICs at 7 days incubation was not observed a significant difference between the effects of allicin and ketoconazole $(\mathrm{p}>0.05)$, but MICs at 10 days incubation, a significant difference was observed $(\mathrm{p} \leq 0.05)$. On the other side, time kill studies revealed that allicin used its fungicidal activity within 12-24 $\mathrm{h}$ of management in vitro as well as ketoconazole. In conclusion, allicin showed very good potential as an antifungal compound against mycoses-causing dermatophytes, almost the same as the synthetic drug ketoconazole. Therefore, this antifungal agent appears to be effective, safe and suitable alternative for the treatment of dermatomycosis.
\end{abstract}

Key words: Allicin, Antifungal drugs, Dermatophytes, MIC (minimal inhibitory concentration.

\section{INTRODUCTION}

Dermatophytosis is among the most popular and prevalent infectious disease worldwide that caused by dermatophytes. Dermatophytes are the main cause of superficial fungal infections of the skin, hair and nails of human and other animals $(2,12)$. Among the various types of dermatophytes, Trichophyton rubrum is clinically the most common genus of human pathogenic dermatophytes and has been the most frequently isolated dermatophytes (20). The azoles group of antifungal drugs has been used to cure dermatomycosis. Ketoconazole and fluoconazole are effective in treating

*Corresponding Author. Mailing address: Department of Medical Biotechnology, School of Advanced Technologies in Medicine, Tehran University of Medical Sciences, Tehran, Iran.; Tel.: +98-9121218439.; E-mail: srezaie@tums.ac.ir 
dermatomycosis but may cause undesirables side effects and toxicity to patients. It has been reported that the increased in the use of these drugs may cause drug resistance to all agents in the azoles group $(1,10)$. Hence, this study was aimed at the investigation on the use of a plant extracts as one of the alternatives to treat dermatomycosis. One of the most common plant extracts being used for medicinal purposes is garlic extracts (Allium sativum). The therapeutic effect of garlic is because of sulfur-containing compounds and thiosulfinates (17). The interaction of sulfur compounds (such as allicin) with sulphur (thiol-containing enzymes) groups of microbial enzymes (such as trypsin and other proteases) which caused inhibition of microbial growth (14). Allicin (diallyl thiosulphinate) is a pure bioactive compound isolated from garlic. It is manufactured via an enzymatic response from freshly crushed garlic (5). Allicin is made with a mixture of allinase (the enzyme that is stocked in an isolated section in garlic) and alliin (a compound in fresh garlic) (14). Allicin has been reported to show antibacterial properties (3) and antifungal activities $(5,10,14,17)$. Ajoene is the ingredient from allicin that has antifungal action against Aspergillus niger and Candida albicans (19). Currently, pure grade allicin is available commercially. Pyun and Shin (10) found out that oil fractions of Allium plants are weaker compared to allicin against Trichophyton spp. The growth of new antifungal agents as well as the incidence of fungal infections in human highlighted the antifungal susceptibility testing of dermatophytes $(6,13)$. The National Committee for Clinical Laboratory Standards (NCCLS) (new name Clinical and Laboratory Standards Institue (CLSI) M38-A established in 2002 has currently issued a standardized in vitro protocol for susceptibility testing. Barros et al. (2) have reported that the susceptibility tests to antifungal agents were suitable and reliable. This method is based on the calculation of the MICs. Pyun and Shin (10) investigated the activity of allicin alone and the combined effects of Allium oils with ketoconazole against Trichophyton spp. They used the broth microdilution method which is based on the calculation of the MIC with further calculation of the FICI for drug combination.

This study was aimed at the evaluation of in vitro antifungal activity of allicin against 10 isolates of T. rubrum, which is determined by broth microdilution.

\section{MATERIALS AND METHODS}

\section{Study design}

Ten isolates of $T$. rubrum were examined for their interactions with natural product; allicin, and drug; ketoconazole respectively. Protocol outlined in Clinical and Laboratory Standards Institue (CLSI, formerly the NCCLS) M38-A, susceptibility testing guidelines for filamentous fungi, was employed (8). These interactions were evaluated in two incubation periods of 7 and 10 days respectively to compare the effectiveness of the treatment.

\section{Isolates}

Nine clinical isolates of T. rubrum from culture collection of clinical isolates preserved at the laboratory of Medical Mycology Department in Tehran University of Medical Sciences, Iran were selected. T. rubrum (ATCC-10218) was used as a control strain. All isolates were kept in sterile saline $(0.85 \%) \mathrm{v} / \mathrm{v} \mathrm{NaCl}$ at $4{ }^{\circ} \mathrm{C}$ until required for bioassays.

\section{Media}

The standard RPMI 1640 (Sigma chemicals Co, USA) medium based on CLSI guidelines M38-A was prepared.

\section{Antifungal compounds and dilutions}

Two antifungal agents namely, allicin (AlexisBiochemicals Co, San Diego, USA) was dissolved in $10 \mathrm{mg} / \mathrm{ml}$ in methanol/water/formic acid (60:40:0.1), then stored at -20 to $-70{ }^{\circ} \mathrm{C}$. Ketoconazole (Sigma chemicals Co, USA), was dissolved in $100 \%$ dimethyl sulfoxide (DMSO) at $1.5 \mathrm{mg} / \mathrm{ml}$ according to the protocol of CLSI M38-A, and arranged as a 
stock solution of $1000 \mu \mathrm{g} / \mathrm{ml}$. The stock was $2 \mathrm{X}$ diluted in RPMI 1640 when the antifungal mixture was examined individually to give the same final strength required for the test (13). A series of twofold dilutions were prepared at 100 times higher than the highest expected final test concentration (intermediate concentration). The DMSO solutions were diluted 1:50 in RPMI 1640 medium to make twice the final intensity required for the test (final concentration). The stock solutions were kept at $-70{ }^{\circ} \mathrm{C}$ until needed. Allicin and ketoconazole drug dillutions ranged from 0.05 to $25.0 \mu \mathrm{g} / \mathrm{ml}$ and 0.03 to $16.0 \mu \mathrm{g} / \mathrm{ml}$, correspondingly, as outlined in the CLSI guidelines M38-A.

\section{Inoculum preparation}

All isolates were transfered from sterile saline $(0.85 \%) \mathrm{v} / \mathrm{v}$ $\mathrm{NaCl}$ solution and subcultured to potato dextrose agar (Difco Laboratories, Detroit, Michigan) and kept at $28^{\circ} \mathrm{C}$ for 7 days to allow for sporulation. The seven-day-old colonies were immersed in $5 \mathrm{ml}$ of sterile saline $(0.85 \%) \mathrm{v} / \mathrm{v} \mathrm{NaCl}$ solution, and the culture exterior was smoothly probed with the tip of a Pasteur pipette to remove the spores. The resulting mixture of conidial and hyphal portions was filtrated with a Whatman's filter no. 40 (pore size: $8 \mu \mathrm{m}$ ), which filtered the hyphal fragments but allowing the passage of microconidia into the collecting flask below as suggested by Santos and Hamdan (12); Santos et al. (13); Barros et al. (2). The conidial suspensions's densities in cuvettes were determined using spectrophotometer (U-1900-UV/VIS, Model: BJO-0003, Hitachi, Tokyo-Japan) at $520 \mathrm{~nm}$ wavelength. The conidial suspensions's densities varied from 0.15 to 0.17 (70\% to $72 \%$ transmittance). The inoculum concentrations ranged from 2$4 \times 10 \varpi$ conidia/ $\mathrm{ml}$. The inoculum quantifications were determined by calculating number of microconidia using a hematocytometer, and by plating $0.01 \mathrm{ml}$ of each inoculum suspensions on sabouraud dextrose agar (Difco Laboratories, Detroit, Michigan). The plates were kept at $28{ }^{\circ} \mathrm{C}$ and monitored daily for the existence of colony from units (CFU).
The CFU were calculated as soon as growth becomes observable. These suspensions were diluted 1:50 in RPMI 1640 medium, which matched with $2 \mathrm{x}$, which was the density needed (about $2-4 \times 10 \tau$ conidia/ $\mathrm{ml}$ ). The test inoculums were prepared in enough volume to inoculate each well with $100 \mu \mathrm{l}$ of the corresponding diluted inoculum suspensions.

\section{Test procedure}

U-bottomed 96 well microdilution plates (Brand 781660, Wertheim, Germany) were used.

\section{Antifungal susceptibility testing}

To each well, $100 \mu l$ of the corresponding fungal inoculum suspension prepared earlier was pipetted and $100 \mu \mathrm{l}$ of the twofold dilution of antifungal compounds. Each test plate included two drug-free controls, one with only RPMI 1640 medium (negative control) and another with $100 \mu \mathrm{l}$ of RPMI 1640 with $100 \mu \mathrm{l}$ of the inoculum suspension (positive control). This medium was prepared by adding powdered RPMI 1640 medium in distilled water, then buffered to PH 7.0 with $0.165 \mathrm{~mol} / \mathrm{L} 3$-[N-morpholino] propane sulfonic acid (MOPS).

\section{Incubation time and temperature}

Microdilution trays were incubated at $28{ }^{\circ} \mathrm{C}$ without agitation and were visually examined after 7 days to observe the growth of colonies as recommended by Santos and Hamdan (12), Santos et al. (13), and Barros et al. (2). After the visual reading, the density of fungal growing on the plates were read with a spectrophotometer (Benchmark plus Microplate Spectrophotometer-Model; Bio-Rad 170-6930-Japan) set at $520 \mathrm{~nm}$ wave length. The absorbance ranged from 0.15 to 0.17 ( 70 to $72 \%$ transmittance) after 7 and 10 days of incubation.

\section{Evaluation of the MIC}

The lowest concentration of an antifungal agent that considerably prevents visible growth of an organism on an agar 
or broth dilution susceptibility test is called MIC which is an acronym for the Minimal Inhibitory Concentration. The results of MIC are recorded in micrograms per milliliter. All growths in the microdilution wells were compared to the growth control to determine the endpoint in microdilution procedures. To compare the actions of the tested drug, the MIC of all drugs were determined. This included the readings of the $\mathrm{MIC}_{50}$ and $\mathrm{MIC}_{90}$; namely, the lowest concentration of the drugs at which 50 or $90 \%$ of the growth of a microorganism was inhibited (9, $11,12,13$, and 15).

\section{Time-kill studies}

Ten isolates of $T$. rubrum were grown as mentioned above in the presence of $12.5 \mu \mathrm{g} / \mathrm{ml}$ allicin and $4 \mu \mathrm{g} / \mathrm{ml}$ ketoconazole to determin the time-kill activities. The reason behind the selection of these concentrations was because they were twofold above the MICs set for these isolates in the microdilution broth method explained above. Conidia in cultures were dislodged after 1, 2, 4, 8, 12 and $24 \mathrm{~h}$ and by scraping and then diluted serially in twofold dilutions which were later poured over sabouraud dextrose agar. Colonies were counted after an interval of 7 days incubation at $28{ }^{\circ} \mathrm{C}$. The calculation of the MFC (Minimal Fungicidal Concentration) as the drug concentration responsible for $99.9 \%$ reduction of viability was made. Such high percentage of reduction took place after 1-24 $\mathrm{h}$ in the presence of allicin and ketoconazole as compared with untreated viable spore counts at $0 \mathrm{~h}$ (14).

\section{RESULTS}

The MICs of allicin was obtained at $0.78-12.5 \mu \mathrm{g} / \mathrm{ml}$ and ketoconazole $0.25-8.0 \mu \mathrm{g} / \mathrm{ml}$ in the study. Results showed that the order of efficacy based on the $\mathrm{MIC}_{50}$ and $\mathrm{MIC}_{90}$ values, all isolates showed almost comparable response to allicin and ketoconazole except for some isolates at $28^{\circ} \mathrm{C}$ at both 7 and 10 days incubation. Values for MICs at 10 days incubation were higher than for 7 days incubation for allicin and ketoconazole (Table 1 and 2).

Time-kill study outcomes reveal that conidia grown from T. rubrum exhibited a reduction in viability after $12-24 \mathrm{~h}$ of incubation in the presence of $12.5 \mu \mathrm{g} / \mathrm{ml}$ allicin and $4 \mu \mathrm{g} / \mathrm{ml}$ ketoconazole (Figure 1). It also shows that allicin decreased the growth of T. rubrum almost as well as ketoconazole.

Table 1. Effects of Allicin and Ketoconazole on Trichophyton rubrum at $28^{\circ} \mathrm{C}$ at 7 days incubation.

\begin{tabular}{lllll}
\hline Dermatophyte isolates & \multicolumn{2}{c}{ Allicin } & \multicolumn{2}{c}{ Ketoconazole } \\
& MIC $_{\mathbf{5 0}} \mathbf{1}^{\mathbf{1}}$ & $\mathbf{M I C}_{\mathbf{9 0}}{ }^{2}$ & $\mathbf{M I C}_{\mathbf{5 0}}$ & $\mathbf{M I C}_{\mathbf{9 0}}$ \\
\hline T. rubrum (1) & 3.125 & 6.25 & 1.0 & 2.0 \\
T. rubrum (2) & 1.56 & 3.125 & 1.0 & 4.0 \\
T. rubrum (3) & 6.25 & 12.5 & 4.0 & 8.0 \\
T. rubrum (4) & 0.78 & 1.56 & 0.25 & 1.0 \\
T. rubrum (5) & 1.56 & 3.125 & 1.0 & 2.0 \\
T. rubrum (6) & 3.125 & 12.5 & 2.0 & 4.0 \\
T. rubrum (7) & 1.56 & 3.125 & 0.5 & 2.0 \\
T. rubrum (8) (ATCC) & 1.56 & 6.25 & 1.0 & 2.0 \\
T. rubrum (9) & 0.78 & 3.125 & 0.5 & 2.0 \\
T. rubrum (10) & 3.125 & 12.5 & 1.0 & 4.0 \\
\hline
\end{tabular}

${ }^{1} \mathrm{MIC}_{50}$ is the MIC at which $50 \%$ of the growth of a microorganism was inhibited $(\mu \mathrm{g} / \mathrm{ml})$.

${ }^{2} \mathrm{MIC}_{90}$ is the MIC at which $90 \%$ of the growth of a microorganism was inhibited $(\mu \mathrm{g} / \mathrm{ml})$. 
Table 2. Effects of Allicin and Ketoconazole on Trichophyton rubrum at $28^{\circ} \mathrm{C}$ at 10 days incubation.

\begin{tabular}{|c|c|c|c|c|}
\hline \multirow[t]{2}{*}{ Dermatophyte isolates } & \multicolumn{2}{|c|}{ Allicin } & \multicolumn{2}{|c|}{ Ketoconazole } \\
\hline & $\mathrm{MIC}_{50}{ }^{1}$ & $\mathrm{MIC}_{90}{ }^{2}$ & $\mathrm{MIC}_{50}$ & MIC $_{90}$ \\
\hline T. rubrum (1) & 3.125 & 6.25 & 1.0 & 2.0 \\
\hline T. rubrum (2) & 3.125 & 6.25 & 2.0 & 8.0 \\
\hline T. rubrum (3) & 6.25 & 12.5 & 4.0 & 8.0 \\
\hline T. rubrum (4) & 1.56 & 6.25 & 0.25 & 1.0 \\
\hline T. rubrum (5) & 3.125 & 6.25 & 1.0 & 2.0 \\
\hline T. rubrum (6) & 3.125 & 12.5 & 2.0 & 4.0 \\
\hline T. rubrum (7) & 1.56 & 6.25 & 1.0 & 2.0 \\
\hline T. rubrum (8) (ATCC) & 3.125 & 12.5 & 1.0 & 2.0 \\
\hline T. rubrum (9) & 1.56 & 6.25 & 0.5 & 2.0 \\
\hline T. rubrum (10) & 6.25 & 12.5 & 2.0 & 4.0 \\
\hline
\end{tabular}

${ }^{1} \mathrm{MIC}_{50}$ is the MIC at which $50 \%$ of the growth of a microorganism was inhibited $(\mu \mathrm{g} / \mathrm{ml})$.

${ }^{2} \mathrm{MIC}_{90}$ is the MIC at which $90 \%$ of the growth of a microorganism was inhibited $(\mu \mathrm{g} / \mathrm{ml})$.

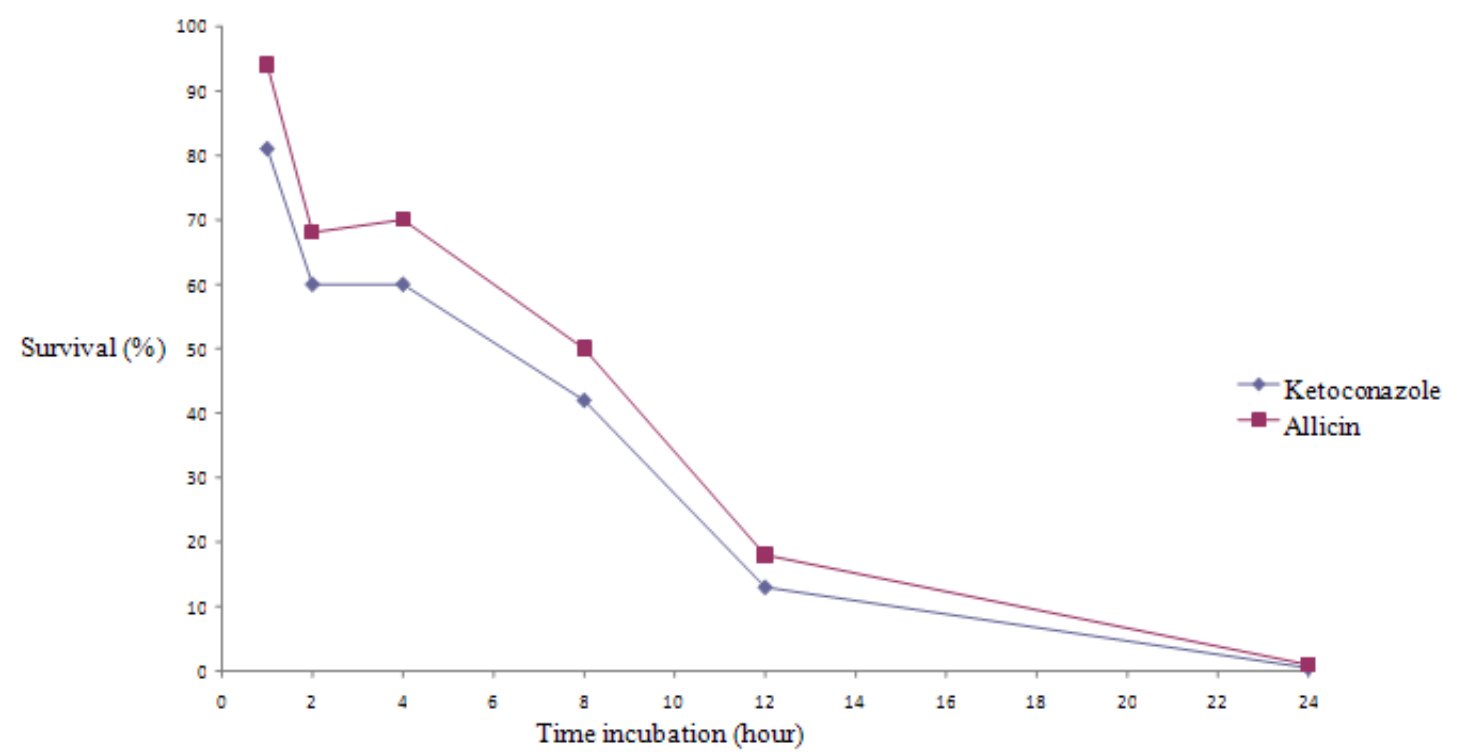

Figure 1. Time-kill curve of T. rubrum (ATCC-10218) conidia incubated in the presence of $12.5 \mu \mathrm{g} / \mathrm{ml} \mathrm{allicin} \mathrm{and} 4 \mu \mathrm{g} / \mathrm{ml}$ keteconazole. The outcomes are characteristic of two independent examinations carried out in triplicate.

\section{Data analysis}

A series of Mann-Whitney tests were used to compare the effects of allicin and ketoconazole on T. rubrum after 7 days incubation. In summary, there is no significant differences between allicin and ketoconazole (Mann-Whitney $\mathrm{U}=28.00$, $\mathrm{p}>0.05)$.

Another series of Mann-Whitney tests were used to compare the effects of allicin and ketoconazole on T. rubrum after 10 days incubation. Briefly, there is significant difference between allicin and ketoconazole (Mann-Whitney $U=18.00$, 
$\mathrm{p} \leq 0.05)$.

Determination of all the MICs were performed in triplicate.

\section{DISCUSSION}

Dermatophytes are a group of fungi able to invade keratinized tissues of human and animals, causing dermatomycosis (2). Dermatophytosis is one of the common infectious diseases worldwide $(2,12)$. The azole antifungal drugs such as imidazole (e.g. ketoconazole) are commonly used in the treatment of dermatomycosis. They are synthetic antifungal drugs and although effective, but, because of increased use of these medications, an incidence of drug resistance to all agents has been reported. They are also known generally to cause side effects such as itching, allergic rash, hepatotoxicity (for ketoconazole) (1). Therefore we investigate the use of a plant-based, biodegradable natural product as an alternative namely allicin. Allicin is a pure, bioactive and the most powerful medicinal compound isolated from garlic and it has different biological properties such as antimicrobial and antifungal activities. Several studies showed that it could be used as the treatment of fungal infections $(3,14)$. This study used allicin as an antifungal agent against 10 isolates of $T$. rubrum in comparison with ketoconazole. The MICs of the allicin and ketoconazole against T. rubrum isolates assessed by broth microdilution assay are listed in Table1 and 2.

Yamada and Azuma (18) proved that the MICs of allicin obtained at $28^{\circ} \mathrm{C}$ for 5 days incubation are $0.78-6.25 \mu \mathrm{g} / \mathrm{ml}$. In our investigation, the MICs of allicin obtained at $28{ }^{\circ} \mathrm{C}$ for 7 days incubation are MIC50 with 0.78-6.25 $\mu \mathrm{g} / \mathrm{ml}$ and MIC90 with 1.56-12.5 $\mu \mathrm{g} / \mathrm{ml}$. Furthermore the MICs of ketoconazole attained at $28{ }^{\circ} \mathrm{C}$ for 7 days incubation are equal to MIC50 and MIC90 of ketoconazole with $0.25-4.0 \mu \mathrm{g} / \mathrm{ml}$ and $0.5-8.0 \mu \mathrm{g} / \mathrm{ml}$ respectively. The results were in line with Santos and Hamdan (12) and Korting et al. (7), but proved to be different from the study of Fernandez-Torres et al. (4).
The results of this study revealed that the MICs for 10 days incubation increased 1 dilutions for drugs tested separately in comparison with the MICs for 7 days incubation except for ketoconazole. The results are supported with Santos and Hamdan (12) who proved that an increased incubation time of 10 days compared to 7 days increases MICs from 1 to 2 dilutions with the same medium, 7 days incubation time compared to 4 days proved to generate similar results.

Therese et al. (16) showed that the isolates were resistant in vitro to ketoconazole, with the $\mathrm{MICs}>0.8 \mu \mathrm{g} / \mathrm{ml}$. Our results displayed that the isolates were all resistance to ketoconazole in MIC except for T. rubrum 4, 7 and 9, thus this data confirm the resistance of T. rubrum isolates to ketoconazole.

Time-kill study outcomes reveal that in the presence of $12.5 \mu \mathrm{g} / \mathrm{ml}$ allicin and $4 \mu \mathrm{g} / \mathrm{ml}$ ketoconazole, conidia grown from T. rubrum exhibited a reduction in viability after $12-24 \mathrm{~h}$ of incubation. It also shows that allicin decreased the growth of T. rubrum almost as well as ketoconazole. Our results are in agreement with the previously study by Shadkchan et al. (14) who demonstrated that conidia grown from strains $A$. fumigatus 13, A. niger 1, A. terreus 3 and A. flavus 2 displayed a loss in viability after $8-12 \mathrm{~h}$ of incubation in the presence of $16 \mu \mathrm{g} / \mathrm{ml}$ allicin. Time-kill study results is also confirmed our MICs results in this study, because both of these results showed that allicin decreased growth of T. rubrum almost as well as ketoconazole.

In conclusion, allicin is potentially as good as antifungal compound against dermatophytes, almost as good as ketoconazole. Therefore, it can be considered as an effective, safe and suitable alternative in the treatment of dermatomycosis. However, further research is required including "in vivo" studies, as there is a lack of data in this field.

\section{REFERENCES}

1. Al-Mohsen, I.; Hughes, WT. (1998). Systemic antifungal therapy: past, 
present and future. Ann Saudi Med. 18 (1), 28-38.

2. Barros, M.; Santos, D.; Hamdan, J. (2007). Evaluation of susceptibility of Trichophyton mentagrophytes and Trichophyton rubrum clinical isolates to antifungal drugs using a modified CLSI microdilution method (M38-A). J Med Microbiol. 56, 514-518.

3. Cai, Y.; Wang, R.; Pei, F.; Liang, B.-B. (2007). Antibacterial Activity of Allicin Alone and in Combination with $b$-Lactams against Staphylococcus spp. and Pseudomonas aeruginosa. J Antibio. 60 (5), 335-338.

4. Ferna'ndez-Torres, B.; Inza, I.; Guarro, J. (2003). Comparison of in vitro antifungal susceptibilities of conidia and hyphae of dermatophytes with thick-wall macroconidia. Antimicrob Agents Chemother. 47, 3371-3372.

5. Gardner, CD.; Lawson, LD.; Block, E.; Chatterjee, LM.; Kiazand, A.; Balise, RR.; Kraemer, HC. (2007). Effect of raw garlic vs. commercial garlic supplements on plasma lipid concentrations in adults with moderate hypercholesterolemia: a randomized clinical trial. Arch Intern Med. 167 (4), 346-353.

6. Ghannoum, M. A.; Chaturvedi, V.; Espinel-Ingroff, A.; Pfaller, M. A.; Rinaldi, M. G.; Lee-Yang, W.; Warnock, D. W. (2004). Intra- and interlaboratory study of a method for testing antifungal susceptibilities of dermatophytes. J Clin Microbiol. 42, 2977-2979.

7. Korting, H. C.; Ollert, M.; Abeck, D.; the German Collaborative Dermatophyte Drug Susceptibility Study Group. (1995). Results of German multicenter study of antimicrobial susceptibilities of Trichophyton rubrum and Trichophyton mentagrophytes strains causing tinea unguium. Antimicrob Agents Chemother. 39, 1206-1208.

8. National Committee For Clinical Laboratory Standards. (2002). Reference method for broth dilution antifungal susceptibility testing of filamentous fungi. Approved standard M38-A. National Committee for Clinical Laboratory Standards, Wayne, Pa.

9. Pelletier, R.; Loranger, L.; Marcotte, H.; Carolis, E. (2002) Voriconazole and fluconazole susceptibility of Candida isolates. J Med Microbiol. 51, 479-483.

10. Pyun, M.; Shin, S. (2006). Antifungal effects of the volatile oils from Allium plants against Trichophyton species and synergism of the oils with ketoconazole. Phytomedicine. 13, 394-400.
11. Rex, JH.; Pfaller, MA.; Walsh, TJ. (2001). Antifungal susceptibility testing: practical aspects and current challenges. Clin Microbiol Rev. 14, 643-658.

12. Santos, D. A.; Hamdan, J. S. (2005). Evaluation of broth microdilution antifungal susceptibility testing conditions for Trichophyton rubrum. J Clin Mirobiol. 43, 1917-1920.

13. Santos, D. A.; Barros, M. E. S.; Hamdan, J. S. (2006). Establishing a Method of Inoculum Preparation for Susceptibility Testing of Trichophyton rubrum and Trichophyton mentagrophytes. J Clin Mirobiol. 44 (1), 98-101.

14. Shadkchan, Y.; Shemesh, E.; Mirelman, D.; Miron, T.; Rabinkov, A.; Wilchek, M.; Osherov, N. (2004). Efficacy of allicin, the reactive molecule of garlic, in inhibiting Aspergillus spp. in vitro, and in a murine model of disseminated aspergillosis. J Antimicrobial Chemother. 53, 832-836.

15. Swinne, D.; Watelle, M.; Nolard, N. (2005). In vitro activities of voriconazole, fluconazole, itraconazole and amphotericin B against non Candida albicans yeast isolates. Rev Iberoam Micol. 22, 24-28.

16. Therese, K. L.; Bagyalakshmi, R.; Madhavan, H. N.; Deepa, P. (2006). Invitro Susceptibility Testing by Agar Dilution Method to Determine The Minimum Inhibitory Concentration of Amphotericin B, Fluconazole and Ketoconazole Against Ocular Fungal Isolates. Indian $\mathrm{J} \mathrm{Med}$ Microbiol. 24 (4), 273-279.

17. Woods-Panzaru, S.; Nelson, D.; McCollum, G.; Ballard, L. M.; Millar, C. (2009). An examination of antibacterial and antifungal properties of constituents described in traditional Ulster cures and remedies. Ulster Med J. 78 (1), 13-15.

18. Yamada, Y.; Azuma, K. (1977). Evaluation of the In Vitro Antifungal Activity of Allicin. Antimicrob Agents and Chemother. 11 (4), 743-749.

19. Yoshida, S.; Kasuga, S.; Hayashi, N.; Ushiroguchi, T.; Matsuura, H.; Shizutoshi, N. (1987). Antifungal Activity of Ajoene Derived from Garlic. Appl Environ Microbiol. 53 (3), 615-617.

20. Zaugg, C.; Monod, M.; Weber, J.; Harshman, K.; Pradervand, S.; Thomas, J.; Bueno, M.; Giddey, K.; Staib, P. (2009). Gene expression profiling in the human pathogenic dermatophyte Trichophyton rubrum during gowth on proteins. Eukaryotic cell. 8 (2), 241-250. 